\title{
الآثار الاقتصادية لسد النهضة الاثيوبي على الميزان المائي المصري والقطاعات المستهلكة للمياه
}

محمد محمد الماحي، ياسمين صلاح عبدالرازق، مها محمد عليوه

قسم الاقتصاد و إدارة الاعمال الزراعية- كلية الزر اعة- جامعة الاسكندرية لهاية

الملخص

نظراً لوجود الكثير من التحديات التي تواجة تتمية وتلبية احتياجات التتمية في القطاعات المستهكة للمباه في مصر، ونظر أ لظهور

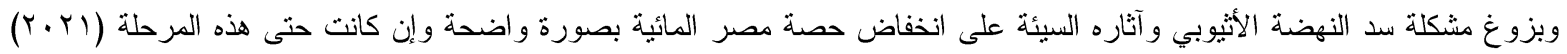

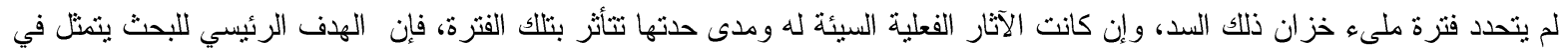

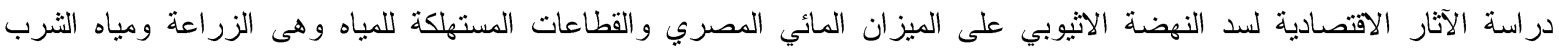
و الصناعة، وقد توصل البحث الى عدة نتائج تتمثل فيما يلي.

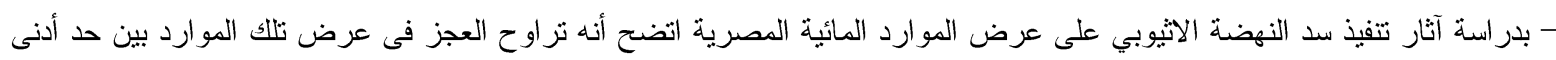

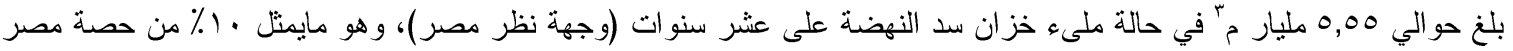

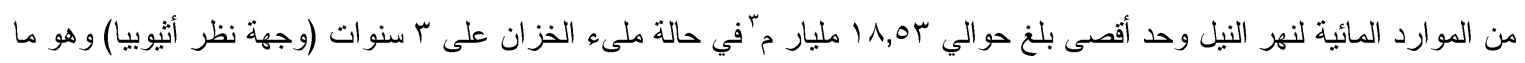

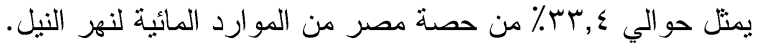

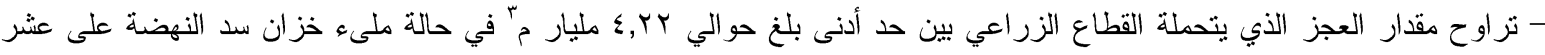

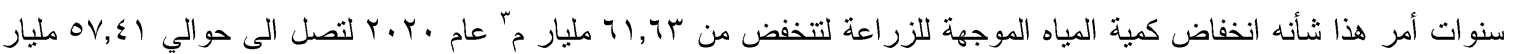

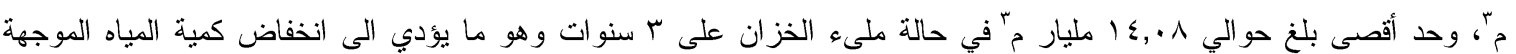

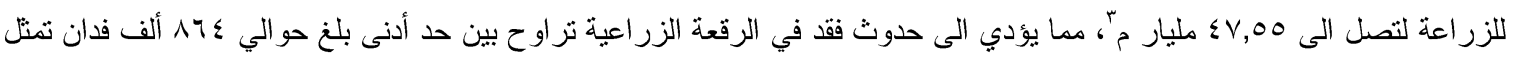

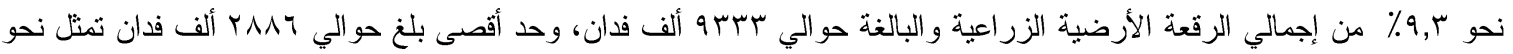

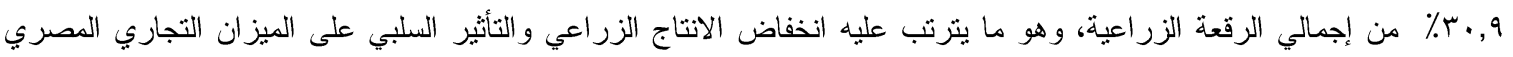

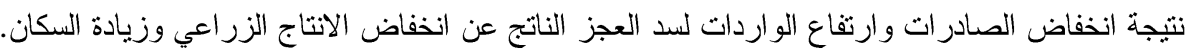

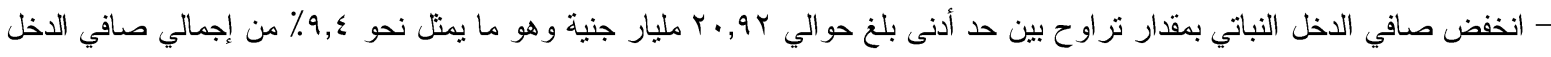

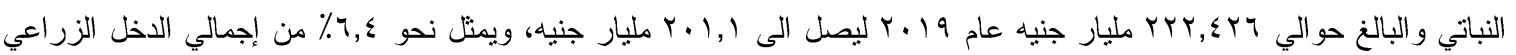

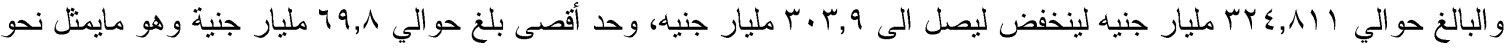

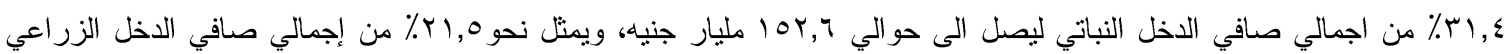

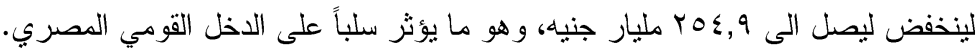

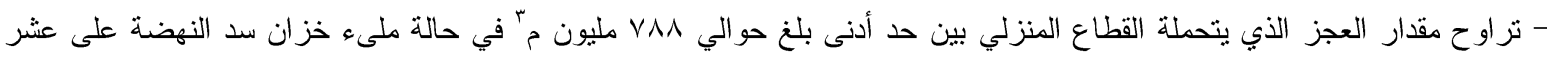

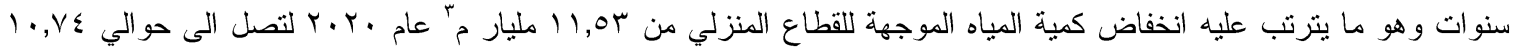

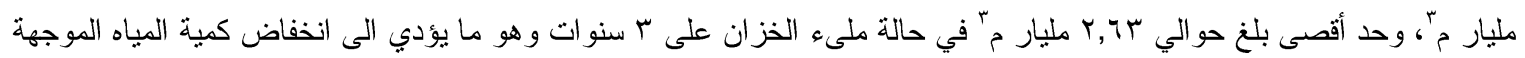

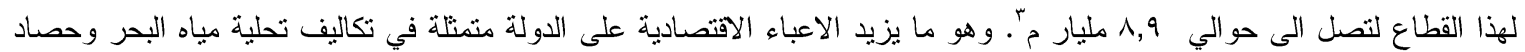

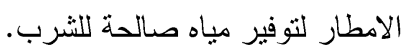

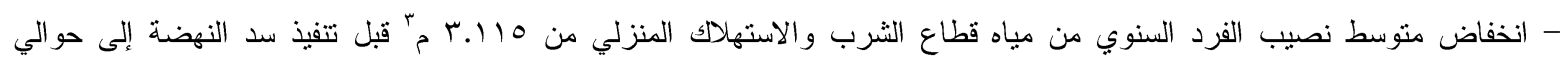

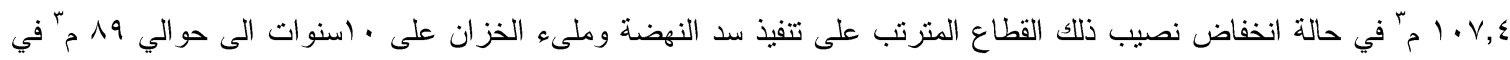

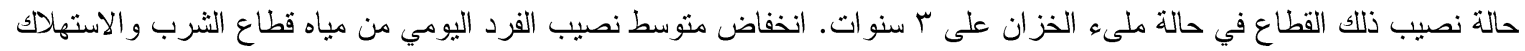

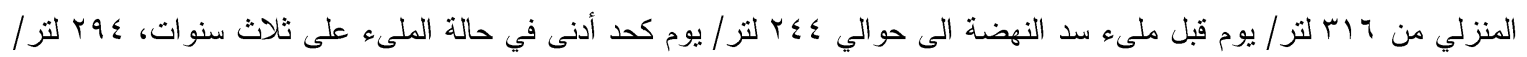
يوم كحد أقصى في حالة الملىه على · ل سنوات. 


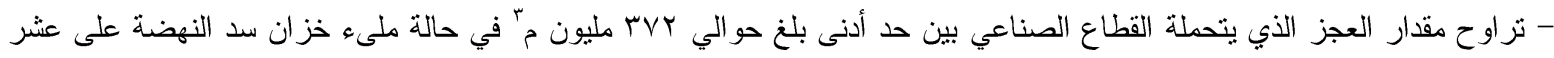

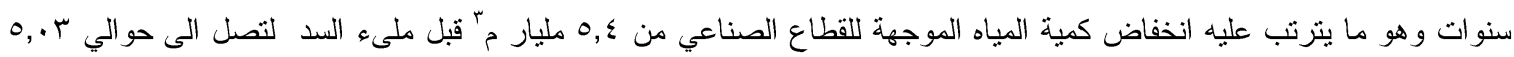

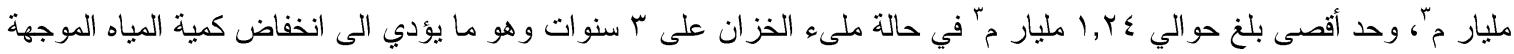

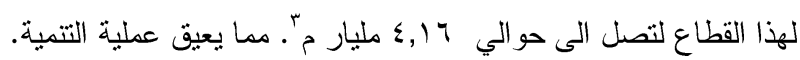
الكلمات المفتاحية: سد النهضة- القطاعات المستهكة للمياه- الميزان المائي.

ومياه الثرب و الصناعة وذلك من خلال دراسة(1)

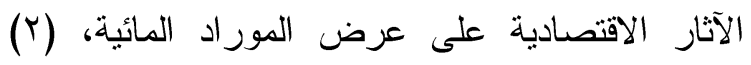

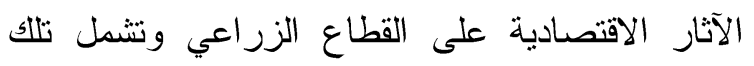

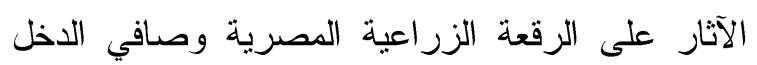

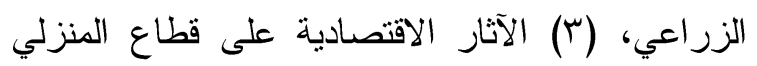

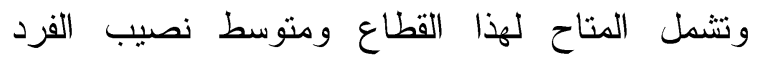

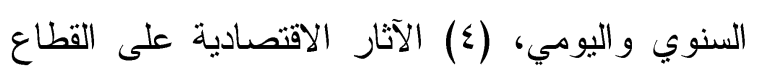
الصناعي. - الصوي و

\section{الأسلوب البحثي ومصادر البيانات}

اعتمد البحث في تحقيق اهدافه على اسلوب التحليل

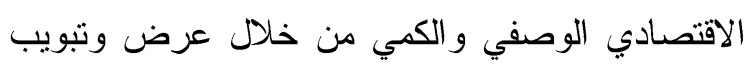
البيانات و المتوسطات الحسابية و الهندسية، ودالة النمو ،

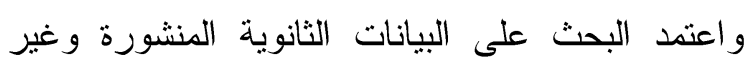
المنشورة الصادرة عن وزارة الزراعة واستصدلاح

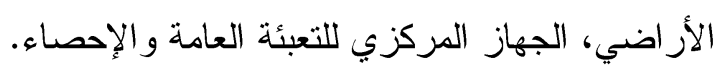

\section{مناقشة النتائج}

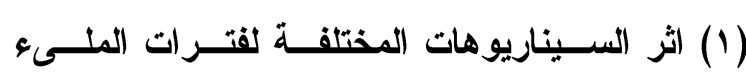
على عرض المور اد المائية:

بدر اسة آثار تتفيذ سد النهضة الاثيوبي على عرض المرليه

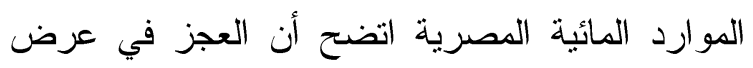
تللك الموارد نزاوح بين حد أدنى بلغ حوالي

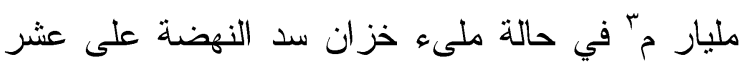

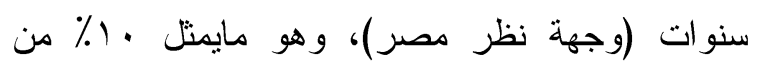

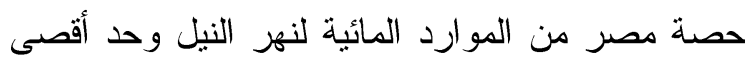

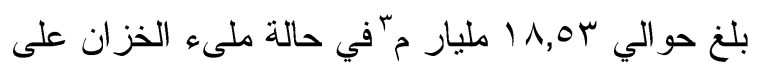

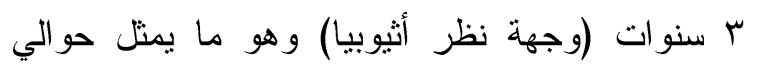

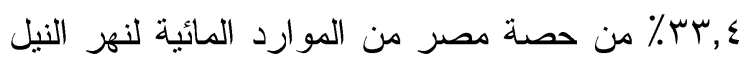

جدول (1). (1)

\section{المقدمة}

يتز ايد الطلب على الموارد المائيــة والــــي يمثـلـل

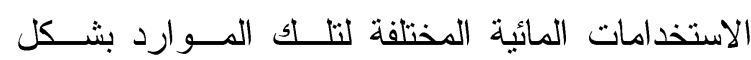

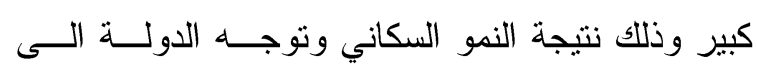

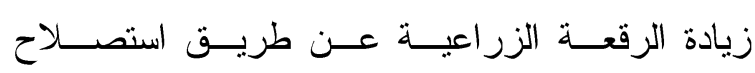

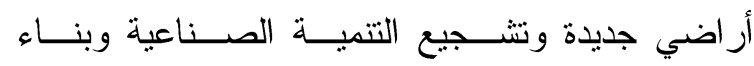

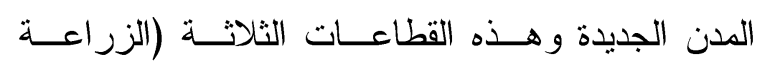

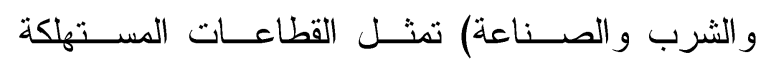

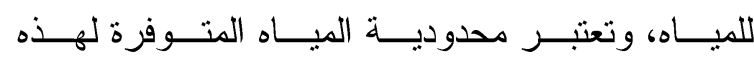

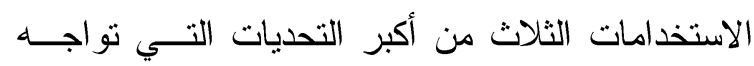
التوسع وتحقيق الهدف المنشود في تلك القطاعات.

\section{المشكلة البحثية}

نظر اً لوجود الكثير مــن التحــديات التــي تواجــة

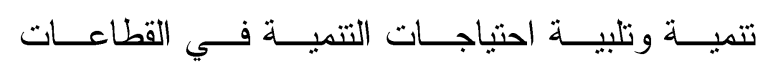

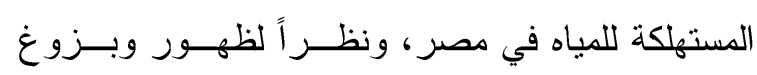

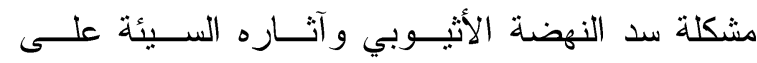

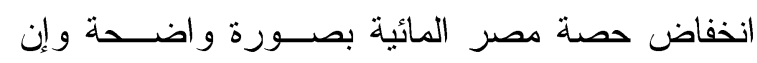

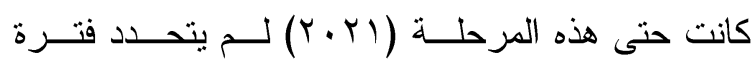

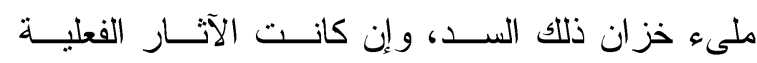

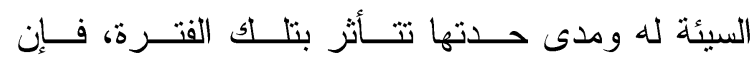

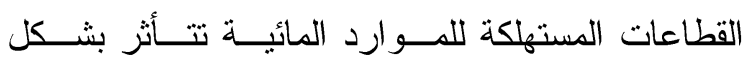

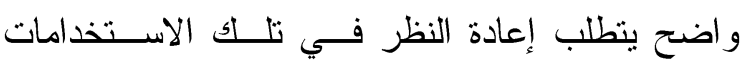
لتخفيف الآثار السيئة عليها وعلـــى التتميــة الثـــاملة المستدامة.

\section{أهداف البحث}

يتمثل الهدف الرئيسي للبحث في دراسة الآثار الاقتصادية لسد النهضة الاثيوبي على الميزان المائي

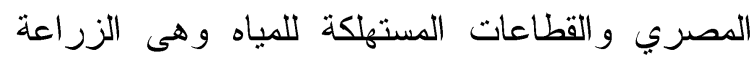


جدول ا: العجز المائي في ضوء أزمة سد النهضة"

\begin{tabular}{|c|c|c|c|}
\hline \multirow{2}{*}{ (من مصر و (السودارن) } & \multicolumn{2}{|c|}{ "العجز المائي(مليار م") } & \multirow[t]{2}{*}{ فترة ملى خزان سد النهضة (سنة) } \\
\hline & السودان & 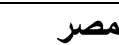 & \\
\hline$r \leqslant, V$ & $7,1 V$ & 11,04 & $r$ \\
\hline 11,0 & $\varepsilon, T Y$ & $1 \Gamma, \wedge \Lambda$ & $\varepsilon$ \\
\hline $1 \varepsilon, \wedge$ & $r, v$. & $11,1$. & 0 \\
\hline$T, r$ & $r, \cdot v$ & $9, Y \mu$ & 7 \\
\hline $1 \cdot, 7$ & $r, 70$ & $v, 90$ & $\mathrm{~V}$ \\
\hline $9, r$ & T,TY & 7,91 & $\Lambda$ \\
\hline$\Lambda, Y$ & $r, .0$ & 7,10 & 9 \\
\hline$V, \varepsilon$ & 1,10 & 0,00 & 1. \\
\hline
\end{tabular}

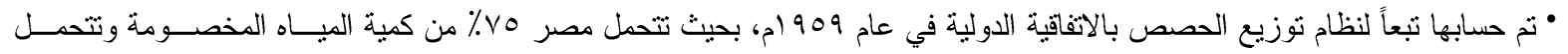

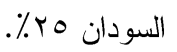

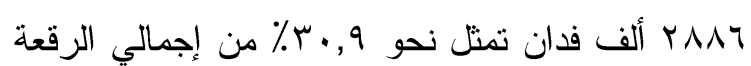

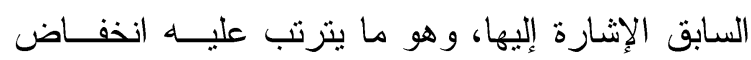

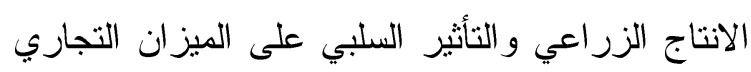

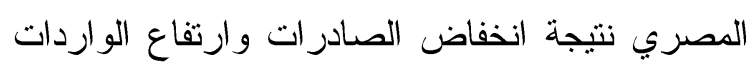

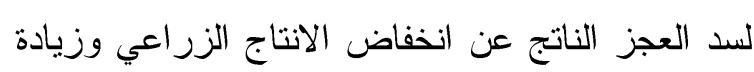

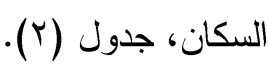

\section{الآثار على إجمالي صافي الاخل الزراعي:}

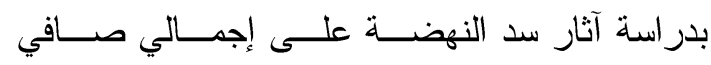

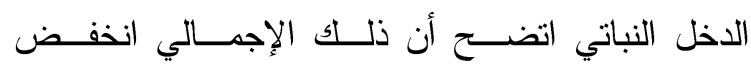

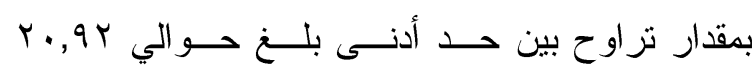

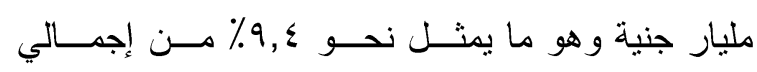

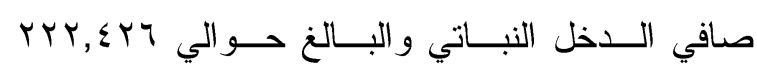

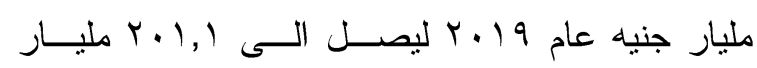

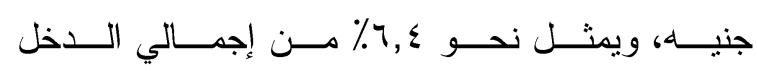

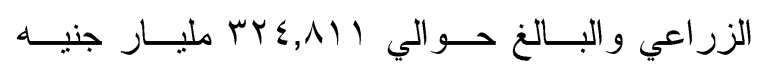

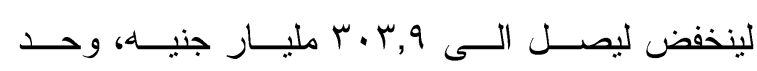

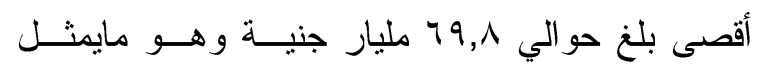

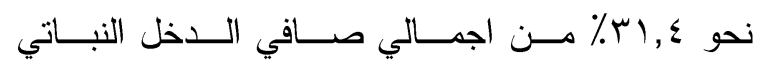

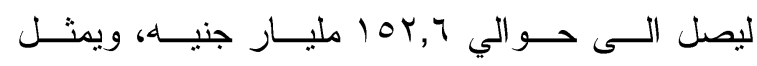

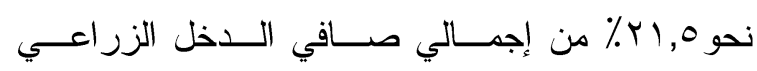

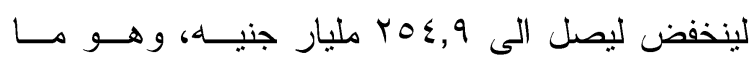

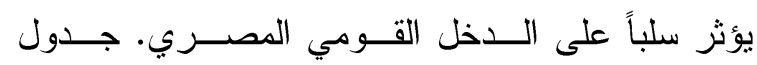

(r) الآثار الاقتصادية لعجز الميزان المائي المصري

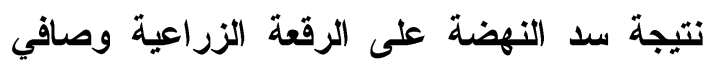
الاخل النباتي. بدر اسة آثار تتفيذ سد النهضية الأثيوبي على القطاع

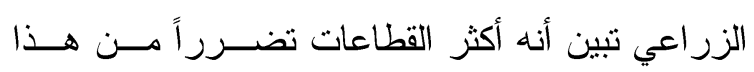

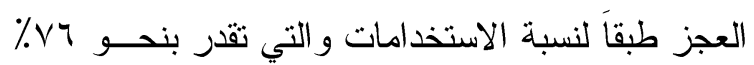

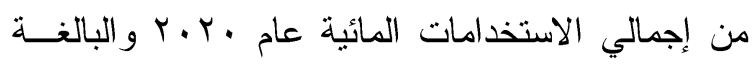

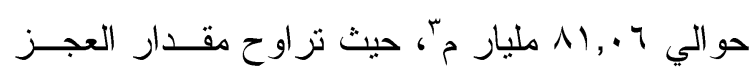

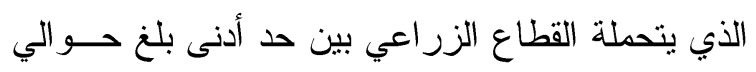

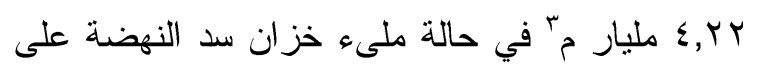

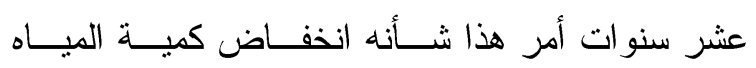

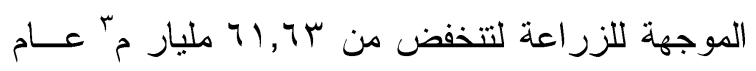

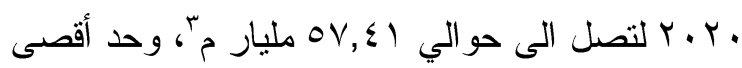

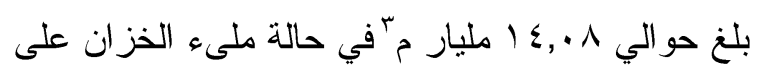
r سنوات وهو ما يؤدي الى انخفــاض كميـــة الميـــاه

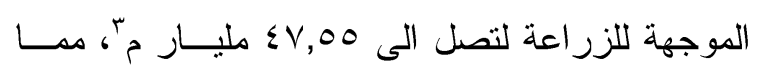
يؤدي الى حدوث فقد في الرقعة الزر اعية تراوح بــين

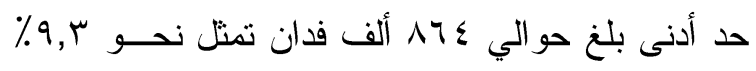

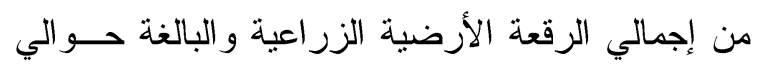

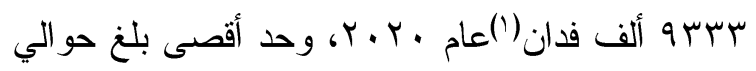
(1) الجهاز المركزي للتعبئة العامة والاحصاء، مصر في ارقام، 
جدول r: الآثار الاتتصادية لعجز الميزان المائي المصري نتيجة سد النهضة على الرقعة الزراعية المصرية.

\begin{tabular}{|c|c|c|c|c|c|}
\hline$\bullet$ & 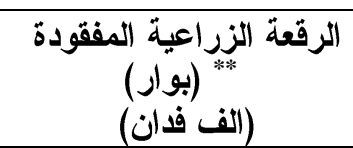 & استهلاك الزراعة بعد & نصيب قطاع الزراعة من & نصيّ مصر مر & فترات الملىه) \\
\hline$r \cdot, 9$ & rANT & $\varepsilon \vee, 00$ & $1 \varepsilon, \cdot \Lambda$ & $\left.0 T_{6}\right) 1$ & $r$ \\
\hline$r T, Y$ & YITY & $01, .1$ & 1,00 & $1 \Gamma, \wedge \wedge$ & $\varepsilon$ \\
\hline 11,0 & IVYq & 04,19 & $\Lambda, \varepsilon \varepsilon$ & 11,1 & 0 \\
\hline $10, \varepsilon$ & $1 \leqslant r V$ & $0 \leqslant, T Y$ & $v, \cdot 1$ & $9, r^{\mu}$ & 7 \\
\hline $1 \pi, r$ & TKMA & 00,09 & $7, \cdot \varepsilon$ & $V, 90$ & v \\
\hline $11, \mathrm{~V}$ & $1 \cdot 1 \mathrm{~V}$ & O4,T & $0, r \cdot$ & 7,91 & $\wedge$ \\
\hline $1 \cdot, r$ & 901 & 07,97 & $\varepsilon, T V$ & 7,10 & 9 \\
\hline $9, r$ & ᄉฯ & $O V, \& 1$ & $\varepsilon, Y Y$ & 0,00 & 1. \\
\hline \multirow{2}{*}{\multicolumn{6}{|c|}{ 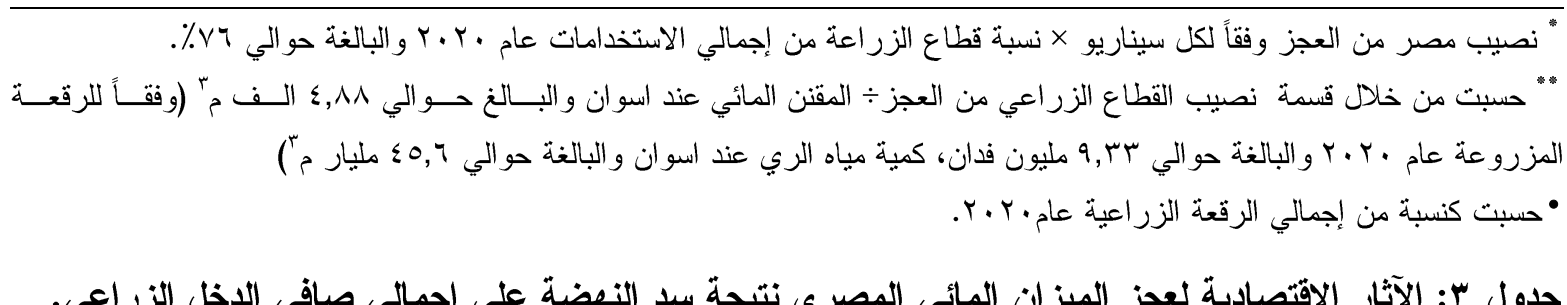 }} \\
\hline & & & & & \\
\hline كي بعد & بعل & $\begin{array}{l}\text { صافي الاخلخاض النباتي } \\
\text { (مليار جنية) }\end{array}$ & 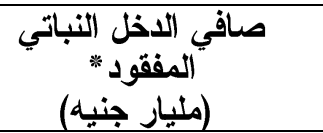 & \multicolumn{2}{|c|}{ فترات الملىء (سنة) } \\
\hline & ros, $9 \vee \leqslant$ & $104,0 \wedge 9$ & $79, \wedge \sim \mathrm{V}$ & & $r$ \\
\hline & $r \vee r, \leqslant 99$ & $1 V \cdot, 11 \leqslant$ & OY,TIY & & $\varepsilon$ \\
\hline & rAY, qVV & $11 ., 09 Y$ & $\varepsilon 1, \wedge \Gamma \varepsilon$ & & 0 \\
\hline & $r q \cdot, . r \varepsilon$ & $1 \wedge \vee, 7 Y q$ & $\Gamma \varepsilon, V \wedge \vee$ & & 7 \\
\hline & $r q \varepsilon, \wedge \leqslant q$ & $19 Y, \leqslant 7 \leqslant$ & rq,97r & & $\mathrm{V}$ \\
\hline & rqA,0.乏 & 197,119 & $r \Psi, r \cdot V$ & & $\Lambda$ \\
\hline & r r & $199, r \leqslant 1$ & Tr,IVA & & 9 \\
\hline & $r \cdot r, \wedge 9 \leqslant$ & $r .1,0.9$ & $r \cdot, 9) V$ & & 1. \\
\hline
\end{tabular}

"حسيت من خلال ضرب الرقعة الزر اعبة المفقودة في متوسط صافي الدخل الفداني السنوي و البالغ حو الي ؟r الف جنيد(قسمة صافي الدخل النباتي † إجمالي الرقعة الزر اعية).

ا ـ القطاع المنزلي ثاني القطاعات تضرراً من العجز الحادث نتيجة ملىء خزان سد النهضة طبقاً لنسبة الاستخدامات والتي تقدر بنحو r,ءء ( من إجمالي

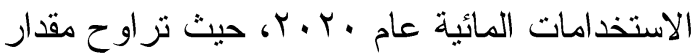

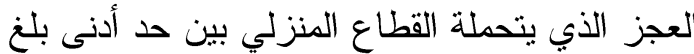

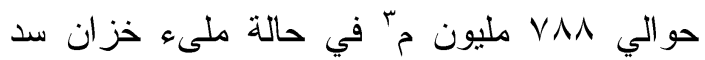
النهضة على عشر سنوات وهو ما يترتب عليه انخفاض كمية المياه الموجهة للقطاع المنزلي من

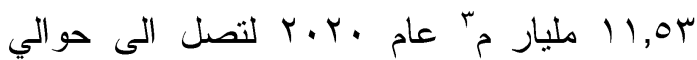

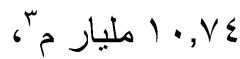

(r) الآثار الاقتصادية لعجز الميزان المائي نتيجة سد النهضة على استهلاك القطاع المنزلي لمياه

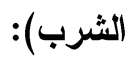
بدراسة الآثار المترتبة على تتفيذ سد النهضة على قطاع استهلاك مياه الشرب في مصر في حالة تحمل لالكل هذا القطاع جزء من العجز المائي المترتب على ذلك و أيضاً في حالة ثبات عدد السكان عند .اء مليون

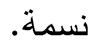
يتضح من خلال البيانات الواردة بجدول (ع) ما يلي: 
جدول ؛ : الآثار المترتبة على القطاع المنزلي في مصر نتيجة ملىء خزان سد النهضة.

\begin{tabular}{|c|c|c|c|c|c|c|}
\hline \multicolumn{2}{|c|}{ 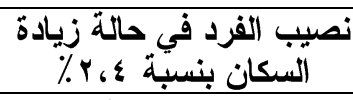 } & \multicolumn{2}{|c|}{ نصيب الفرد في حالةً ثبات } & \multirow{2}{*}{ المنزلي بعد الخصلّم الفطاع } & \multirow{2}{*}{ 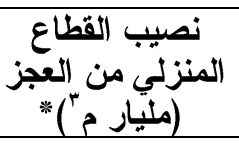 } & \multirow[t]{2}{*}{ فنترة الملى؛ } \\
\hline لتز/يوم & السنوي & لتر/يوم & السنوي (م") & & & \\
\hline$r .1$ & $11 Y, 7$ & MI & $110, r$ & $11,0 \mathrm{~T}$ & & قبل فترة الملىء \\
\hline THA & 17,9 & $r \leqslant \varepsilon$ & $19,$. & $\wedge, q$. & $r, 4 \pi$ & $r$ \\
\hline Yoy & $9 \pi, \varepsilon$ & YTY & 90,7 & 9,07 & $1,9 \mathrm{~V}$ & $\varepsilon$ \\
\hline ry4 & $9 V, r$ & TVT & 99,0 & 9,90 & 1,01 & 0 \\
\hline TVT & 99,1 & rA. & $1 \cdot r, Y$ & $1 \cdot, Y Y$ & $1, r)$ & 7 \\
\hline rVA & $1 \cdot 1,7$ & r10 & $1 \cdot \varepsilon,$. & $1 \cdot, \varepsilon$ & $1,1 \pi$ & v \\
\hline TAY & $1 . r, q$ & r^q & $1.0, \varepsilon$ & $1,0 \leqslant$ &., 99 & $\Lambda$ \\
\hline r 10 & $1 \cdot \varepsilon, 1$ & rar & $1.7,7$ & $1 \cdot, 74$ & $\cdot, \mathrm{AV}$ & 9 \\
\hline YAV & $1 \cdot \leq, 9$ & rqs & $1 \cdot v, \varepsilon$ & $1 \cdot, v \varepsilon$ & $\cdot, \vee \wedge \wedge$ & 1. \\
\hline
\end{tabular}

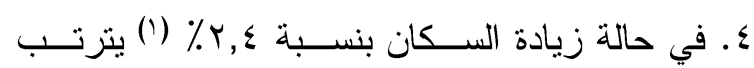

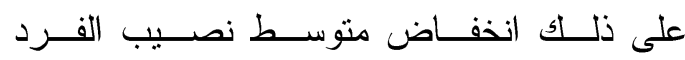

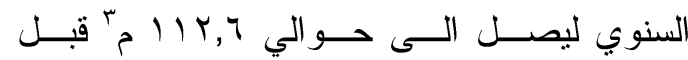

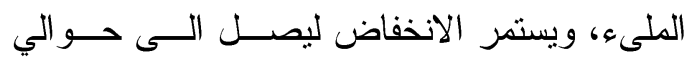

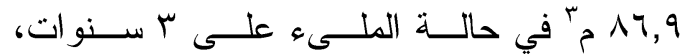

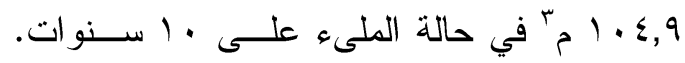

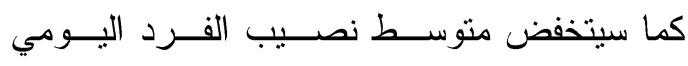

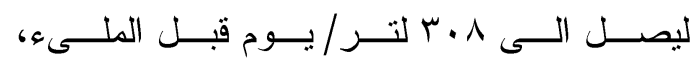

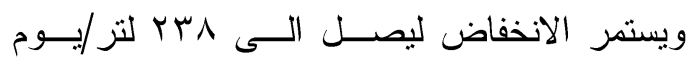

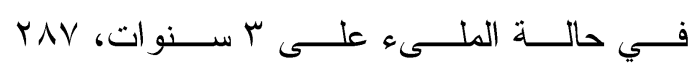

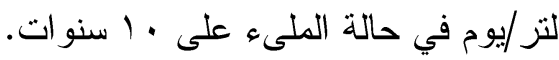

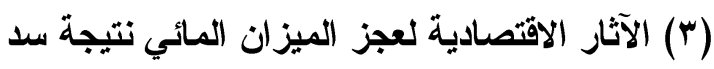
النهضة على استهلاك القطاع الصناعي:

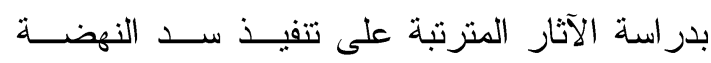

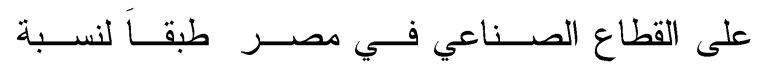

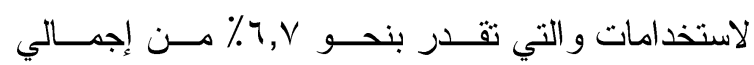

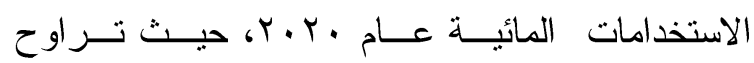

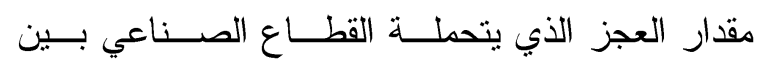

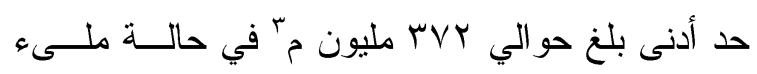

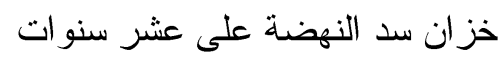

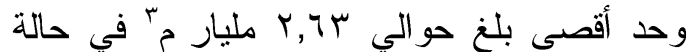

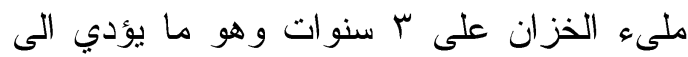
انخفاض كمية المياه الموجهة لهذا القطاع لتصل ملى لهرئ

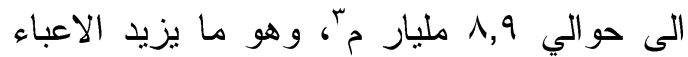
الاقتصادية على الدولة متمتلة في تكاليف تحلية مياه البحر وحصاد الامطار لتوفير مياه صالحة

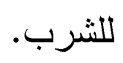
r. انخفاض متوسط نصيب الفرد السنوي من مياه قطاع

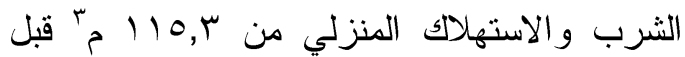

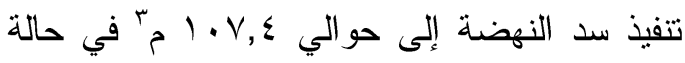
انخفاض نصيب ذلك القطاع المترتب على تتفيذ سد

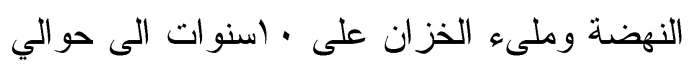

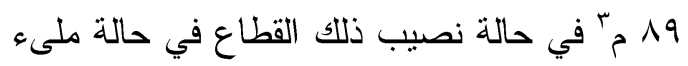

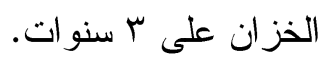
r. انخفاض متوسط نصيب الفرد اليــومي مــن ميــاه

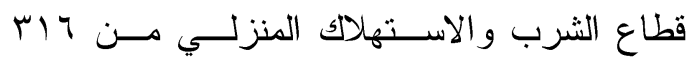

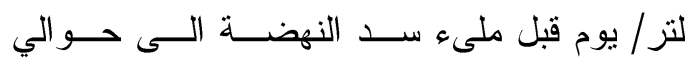

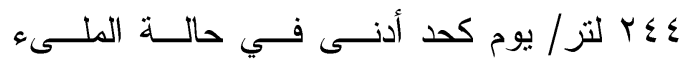

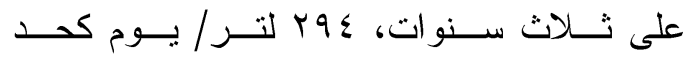
أقصى في حالة الملىء على • ( سنو ات. 
جدول ه: الآثار المترتبة على القطاع الصناعي في مصر نتيجة ملىء خزان سد النهضة.

\begin{tabular}{|c|c|c|c|}
\hline استهلاك القطاع الصناعي بعد & نصيب القطاع الصناعى العزئ & نصيب مصن العزي & فترة الملىء (سنـة) \\
\hline $0, \Sigma$ & & & قبل فتزة الملىء \\
\hline$\varepsilon, 17$ & $1, r \varepsilon$ & 11,04 & $r$ \\
\hline$\varepsilon, \varepsilon V$ & $\cdot, 9 \pi$ & $1 \Gamma, \wedge \Lambda$ & $\varepsilon$ \\
\hline$\varepsilon, 77$ & $\cdot, \mathrm{V} \leqslant$ & 11,1 & 0 \\
\hline$\varepsilon, \vee \wedge$ & זT, & $9, r T$ & 7 \\
\hline$\varepsilon, \wedge V$ &., 04 & $V, 90$ & V \\
\hline$\varepsilon, 9 \pi$ & $\cdot, \leqslant \mathrm{V}$ & 7,91 & $\Lambda$ \\
\hline$\varepsilon, 99$ & $\cdot, \varepsilon 1$ & 7,10 & 9 \\
\hline $0, \cdot r$ & $\cdot, \Gamma V Y$ & 0,00 & 1. \\
\hline
\end{tabular}

محمد حافظ الماحي، ياســمين صــلاح عبــدالرازق، محمد علي فتح الله، التوجيه الاقتصـــادي للمـــوارد

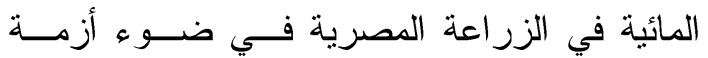

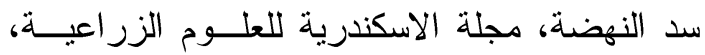

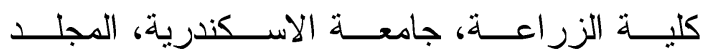

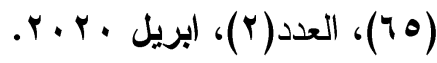

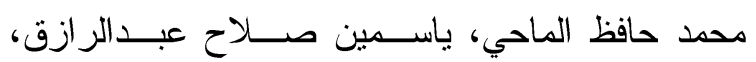
محمد احمد سلطان، دور تطبيق بعـض الجوانـبـ

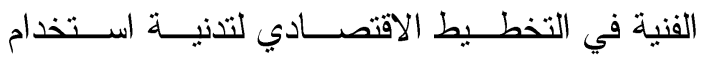

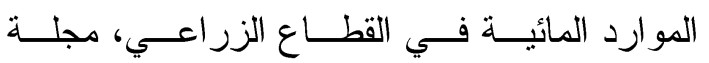

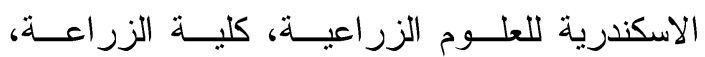

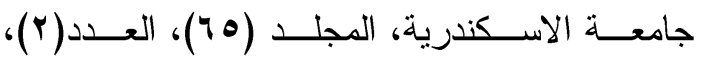

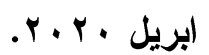
محمد حافظ الماحي، ياســمين صــلاح عبــدالرازق،

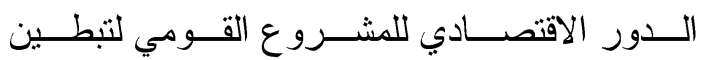

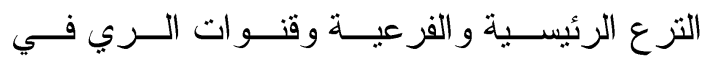

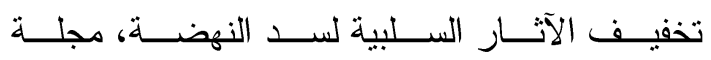

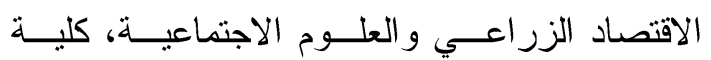

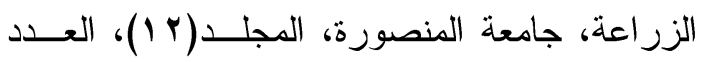

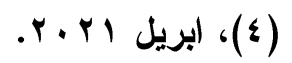

وهو ما يترتب عليه انخفاض كميــة الميــاه الموجهــة

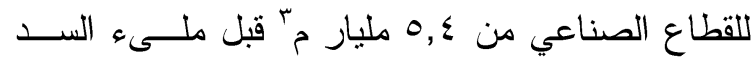

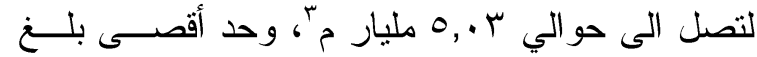

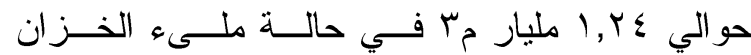
على ب سنوات وهو ما يؤدي الـــى انخفــاض كميــة

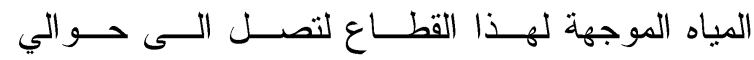

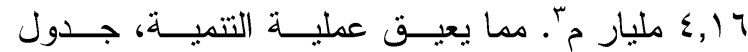

\section{المراجع}

الجهاز المركزي للتعبئة العامة والاحصـاء، مصر فـي

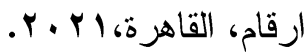
الجهاز المركزى للتعبئة العامـــة و الإحصـــاء، النشــرة

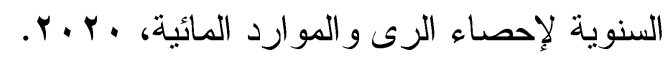




\title{
The Economic Effects of the Ethiopian Renaissance Dam on the Water Balance Egyptian and Water-Consuming Sectors
}

\author{
Mohamed Elmahy, Yasmen Salah Abd El Razek, Maha Mohamed Aliwa \\ Department of Economics and Agribusiness, Faculty of Agriculture, Alexandria University
}

\begin{abstract}
In view of the many challenges facing the development and meeting the development needs in the waterconsuming sectors in Egypt, and due to the emergence and emergence of the problem of the Ethiopian Renaissance Dam and its negative effects on the clear decrease in Egypt's water share, although until this stage (2021) the period of filling the reservoir of that dam has not been determined, And if the actual negative effects of it and the extent of its severity affected by that period, the main objective of the research is to study the economic effects of the Ethiopian Renaissance Dam on the Egyptian water balance and the water-consuming sectors, which are agriculture, drinking water and industry, and the research reached several results, which are as follows.

By studying the effects of the implementation of the Grand Ethiopian Renaissance Dam on the supply of Egyptian water resources, it became clear that the deficit in the supply of these resources ranged between a minimum of about 5.55 billion $\mathrm{m} 3$ in the case of filling the reservoir of the Renaissance Dam over ten years (Egypt's view), which represents $10 \%$ of Egypt's share of the Nile River's water resources reached a maximum of about 18.53 billion $\mathrm{m} 3$ in the case of filling the reservoir over 3 years (Ethiopia's point of view), which represents about $33.4 \%$ of Egypt's share of the Nile's water resources.

The amount of the deficit borne by the agricultural sector ranged from a minimum of about 4.22 billion $\mathrm{m} 3$ in the case of filling the reservoir of the Renaissance Dam over ten years. This would lead to a decrease in the amount of water destined for agriculture to drop from 61.63 billion $\mathrm{m} 3$ in 2020 to reach about 57.41 billion $\mathrm{m}$ 3 , and a maximum of about 14.08 billion $\mathrm{m} 3$ in the case of filling the reservoir over 3 years, which leads to a decrease in the amount of water destined for agriculture to reach 47.55 billion $\mathrm{m} \mathrm{3}$, which leads to a loss of agricultural land ranging from a minimum of about 864 One thousand, representing about $9.3 \%$ of the total agricultural land, amounting to about 9333 thousand, and a maximum of about 2,886 thousand, representing about $30.9 \%$ of the total agricultural area, which results in a decrease in agricultural production and a negative impact on the trade balance This is due to the decrease in exports and the increase in imports to fill the deficit resulting from the decrease in agricultural production and the increase in population.

Net plant income decreased by a minimum of about 20.92 billion pounds, representing about $9.4 \%$ of total net plant income of about EGP 222,426 billion in 2019 to EGP 201.1 billion, representing about $6.4 \%$ of total agricultural income of ABOUT EGP 324,811 billion to fall to EGP 303.9 billion, A maximum of 69.8 billion pounds, representing about $31.4 \%$ of total net plant income to reach about EGP 152.6 billion, represents about $21.5 \%$ of total net agricultural income to fall to EGP 254.9 billion, which negatively affects Egypt's national income.

The amount of the deficit borne by the domestic sector ranged from a minimum of about 788 million $\mathrm{m} 3$ in the case of filling the reservoir of the Renaissance Dam over ten years, which resulted in a decrease in the amount of water directed to the domestic sector from 11.53 billion m3 in 2020 to about 10.74 billion m3 And a maximum of about 2.63 billion $\mathrm{m} 3$ in the case of filling the reservoir over 3 years, which leads to a decrease in the amount of water destined for this sector to reach about 8.9 billion $\mathrm{m} 3$. This increases the economic burdens on the state represented in the costs of desalinating sea water and harvesting rain to provide potable water.

The amount of deficit borne by the industrial sector ranged between a minimum of about 372 million $\mathrm{m} 3$ in the case of filling the reservoir of the Renaissance Dam over ten years, which resulted in a decrease in the amount of water destined for the industrial sector from 5.4 billion $\mathrm{m} 3$ before filling the dam to reach about 5.03 billion $\mathrm{m} 3$, and a maximum of about 1.24 billion $\mathrm{m} 3$ in the case of filling the reservoir over 3 years, which leads to a decrease in the amount of water destined for this sector to reach about 4.16 billion $\mathrm{m} 3$. which hinders the development process.
\end{abstract}

